\title{
Intelligent resource management for 5G
}

\author{
Zhi Liu ${ }^{1} \cdot$ Qiang $\mathrm{Liu}^{2} \cdot$ Ryan Shea ${ }^{3} \cdot$ Wei Cai ${ }^{4} \cdot$ Zehua Wang $^{5} \cdot$ Yongyi Ran $^{6}$
}

Published online: 11 February 2020

(C) Springer Science+Business Media, LLC, part of Springer Nature 2020

Nowadays, the Internet of things (IoT) connects the physical devices, vehicles, home appliances and other items embedded with electronics, software, sensors, actuators with connectivity such as WiFi, ZigBee, NBIoT and cellular networks. The supported ubiquitous information exchange and content sharing among the smart devices enable numerous applications such as mobile social services, crowdsourcing, mobile robot in order to realize a smarter city, grid, health, and more intelligent transportation systems. On the other hand, these newly emerging applications bring great challenges to the current wireless network in various aspects such as the delay and throughput. The fifth-generation network (5G) is emerging as a promising solution to meet the requirements of explosive data traffic in future wireless and mobile communications, and will be deployed soon. The opportunities and inherent challenges of $5 \mathrm{G}$ rapidly gain great attention from academics, industries, and governments.

Zhi Liu
liu@ieee.org
Qiang Liu
qiangliu06@nudt.edu.cn
Ryan Shea
ryan_shea@sfu.ca
Wei Cai
vcaiwei@ cuhk.edu.cn
Zehua Wang
zwang@ece.ubc.ca
Yongyi Ran
yyran@ntu.edu
1. Shizuoka University, Hamamatsu, Japan
$2 \quad$ National University of Defense Technology, Changsha,
China
3 Simon Fraser University, Burnaby, Canada
$4 \quad$ The Chinese University of Hong Kong, Shenzhen, China
$5 \quad$ The University of British Columbia, Vancouver, Canada
$6 \quad$ Nanyang Technological University, Singapore, Singapore

This special issue encourages innovative papers that advance the 5G applications, and aims to attract research contributions that address the major opportunities and challenges in novel intelligent resource management for $5 \mathrm{G}$ with the emphasis on both new analytical outcomes and novel application scenarios. Finally, we have 9 paper published after the peer-review process.

The first paper "A VDTN Scheme with Enhanced Buffer Management" by Zhaoyang Du, et al. provided a vehicular delay tolerant networks routing scheme, which combined the message deliver strategy of PRoPHET protocol, the message copy control strategy of Spray-and-Wait protocol and an enhanced buffer management scheme, where the buffer management scheme was designed to improve certain network performance goals, namely, maximizing the average delivery ratio and minimizing the average delivery delay. The simulation results showed that the proposed scheme results in better system performance than the existing baseline routing protocols.

The second paper "Enabling 5G: Sentimental Image Dominant Graph Topic Model for Cross-modality Topic Detection" by Jiayi Sun, et al. proposed a sentimental image dominant graph topic model, which can detect the topic from the heterogeneous data and mine the sentiment of each topic, with the aim aims to help make the $5 \mathrm{G}$ network and its applications more intelligently.

The third paper "Location-Partition-Based Channel Allocation and Power Control Methods for C-V2X Communication Networks" by Ping Wang, et al., studied the resource allocation (RA) problem in a vehicle-to-vehicle (V2V) communication network when the in-band deviceto-device (D2D) technology was applied to support cellular-based vehicle-to-everything (CV2X) communication. The proposed scheme greatly outperformed the conventional solutions.

The fourth paper "Increasing network throughput based on dynamic caching policy at wireless access points" by Jianyi Ren, et al. considered the cache hit probability in edge wireless APs caching, and used 0-1 knapsack problem dynamic programming to maximize the local cache-hit probability of wireless AP. 
The fifth paper "Cooperative Caching and Delivery Algorithm Based on Content Access Patterns at Network Edge" by Lintao Yang, et al. proposed cooperative caching based on user access patterns at the network edge. Tensor decompositions of multi aspect data were applied. Furthermore, the authors designed a content placement algorithm that simultaneously considered the hit ratio and the cooperative cost. In every cooperative caching domain, the contents that frequently interacted with the BSs in the domain were placed and shared accordingly. The simulation results based on the real dataset also revealed the superiority of the proposed scheme.

The sixth paper "Traffic Big Data Assisted V2X Communications toward Smart Transportation" by Chang An and Celimuge $\mathrm{Wu}$, first gave a review on the recent research efforts for solving communication challenges in vehicular networks, and then proposed a traffic Big Data Assisted Communication scheme, BDAC, for vehicular networks. The proposed scheme used past traffic big data to estimate the vehicle density and velocity, and then used the prediction results to improve the $\mathrm{V} 2 \mathrm{X}$ communications. The authors implemented the proposed scheme in a multi hop broadcast protocol to show the advantage of the proposed scheme by comparing with other baselines.

The seventh paper "Multi-Objective Computation Offloading for Internet of Vehicles in Cloud-Edge Computing”, by Xiaolong $\mathrm{Xu}$, et al., proposed a cloud-edge computing-enabled computation offloading method. The proposed scheme was verified by the simulations and the effectiveness was observed from the simulation results.

The eighth paper "A Lightweight Heterogeneous Network Clustering Algorithm Based on Edge Computing for $5 G$ " by Ruizhong Du, et al., proposed and evaluated an improved clustering algorithm for energy conservation in $5 \mathrm{G}$ heterogeneous networks called LEC-SEP, which was used to organize edge devices in edge computing and select $\mathrm{CH}$ for data aggregation. The simulation results showed that the proposed algorithm can effectively extend the stability period and transmit more data when it is less affected by the fluctuation of the number of cluster head.

The ninth paper "Research on Unlicensed Spectrum Access Mechanism Based on Reinforcement Learning in LAA/WLAN Coexisting Network" firstly introduced an access mechanism based on reinforcement learning after making the summery of the existing coexistence mechanisms. This mechanism took Listen Before Talk (LBT) mechanism as a technical basis to have adaptive adjustment of the channel access probability, thus to increase the system capacity. Meanwhile, the system's throughput was processed using a proportional fair utility function, and utility value was considered as the return value of the system to ensure fairness between the LAA and the WLAN. Simulation results indicated that the proposed algorithm can effectively maximize the system throughput.

Publisher's Note Springer Nature remains neutral with regard to jurisdictional claims in published maps and institutional affiliations. 